\title{
Signatures of Intra- and Intermolecular Vibrational Coupling in Halogenated Liquids Revealed by Two-Dimensional Raman-Terahertz Spectroscopy
}

\author{
Ciardi, Gustavo ; Berger, Arian ; Hamm, Peter ; Shalit, Andrey
}

\begin{abstract}
Hybrid 2D Raman-THz spectroscopy with the Raman-THz-THz (RTT) pulse sequence is used to explore the ultrafast intra- and intermolecular degrees of freedom of liquid bromoform $(\mathrm{CHBr} 3)$ in the frequency range of 1-8 THz. Cross peaks observed in these $2 \mathrm{D}$ spectra are assigned to the coupling between the narrow intramolecular modes of the molecules and the much broader intermolecular degrees of freedom of the liquid. This assignment is based on the frequency position of the crosspeaks, however, it is shown that these frequency positions can be deduced accurately only when properly taking into account the convolution of the molecular response with the instrument response function of the experimental setup, the latter of which distorts the 2D spectra considerably. The assignment is backed up with additional experiments on diiodomethane (CH2I2), which has only one intramolecular mode in the frequency range of the experiment, and hence excludes the possibility of intramolecular couplings.
\end{abstract}

DOI: https://doi.org/10.1021/acs.jpclett.9b01528

Posted at the Zurich Open Repository and Archive, University of Zurich

ZORA URL: https://doi.org/10.5167/uzh-183161

Journal Article

Accepted Version

Originally published at:

Ciardi, Gustavo; Berger, Arian; Hamm, Peter; Shalit, Andrey (2019). Signatures of Intra- and Intermolecular Vibrational Coupling in Halogenated Liquids Revealed by Two-Dimensional Raman-Terahertz Spectroscopy. Journal of Physical Chemistry Letters, 10(15):4463-4468.

DOI: https://doi.org/10.1021/acs.jpclett.9b01528 


\title{
Signatures of Intra-/Intermolecular Vibrational Coupling in Halogenated Liquids Revealed by 2D Raman-THz Spectroscopy
}

\author{
Gustavo Ciardi, Arian Berger, Peter Hamm* and Andrey Shalit \\ Department of Chemistry, University of Zurich, Winterthurerstrasse 190, \\ CH-8057Zurich, Switzerland, *peter.hamm@chem.uzh.ch
}

\begin{abstract}
Hybrid 2D Raman-THz spectroscopy with the Raman-THz-THz (RTT) pulse sequence is used to explore the ultrafast intra- and intermolecular degrees of freedom of liquid bromoform $\left(\mathrm{CHBr}_{3}\right)$ in the frequency range of $1-8 \mathrm{THz}$. Cross peaks observed in these $2 \mathrm{D}$ spectra are assigned to the coupling between the narrow intramolecular modes of the molecules and the much broader intermolecular degrees of freedom of the liquid. This assignment is based on the frequency position of the crosspeaks, however, it is shown that these frequency positions can be deduced accurately only when properly taking into account the convolution of the molecular response with the instrument response function of the experimental setup, the latter of which distorts the 2D spectra considerably. The assignment is backed up with additional experiments on diiodomethane $\left(\mathrm{CH}_{2} \mathrm{I}_{2}\right)$, which has only one intramolecular mode in the frequency range of the experiment, and hence excludes the possibility of intramolecular couplings.
\end{abstract}

Table of Content Figure:

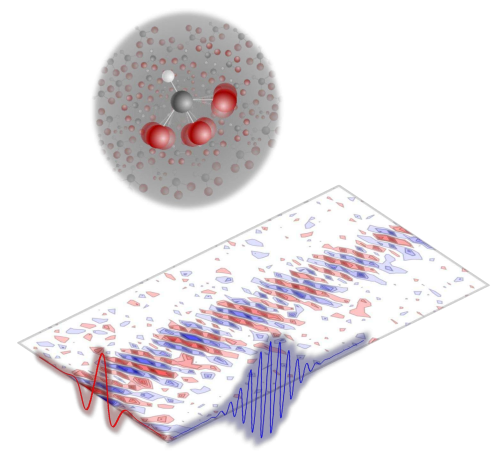

The spectral properties of liquids in the Terahertz $(\mathrm{THz})$ frequency range reflect the thermally populated $\left(\leq k_{B} T=200 \mathrm{~cm}^{-1}\right)$ intermolecular degrees of freedom, such as, for example, hydrogen bonds and hindered rotation which are ultimately responsible for the bulk properties of liquids and processes like solvation. ${ }^{1,2}$ It is, however, challenging to follow and monitor such properties in detail due to the, generally, ultrafast timescales at which they occur. In one-dimensional spectra, fast dephasing and the large anharmonicity of the modes leads to a significant broadening of any spectral feature, both in Raman $^{3-5}$ and $\mathrm{THz}$ spectroscopy ${ }^{6,7}$ limiting the amount of information that is possible to extract. Multidimensional spectroscopies turned out to be a powerful tool to partly circumvent this problem by spreading the spectra along multiple frequency axes; well-known methods as NMR spectroscopy and 2D infrared spectroscopy have demonstrated this very concept with the help of devised pulse sequences, successfully managing to retrieve detailed structural information and broadening mechanism of nuclear spins ${ }^{8}$ and intra-molecular vibrations. ${ }^{9-11}$

Along the same line, fifth-order two dimensional Raman spectroscopy and 2D-THz spectroscopy were introduced, in order to extend the above-mentioned con- cepts to the low-frequency regime. ${ }^{12}$ The former, theoretically proposed by Tanimura and Mukamel, ${ }^{13}$ relies on a 5th order non-linear signal that needs to be isolated from the much stronger third order cascading background, making the experimental realization extremely difficult. ${ }^{14-18}$ Only recently, schemes using a pulse shaper have overcome this issue. ${ }^{19} 2 \mathrm{D}-\mathrm{THz}$ experiments, conceptually the natural extension of 2D-IR to low-frequencies, have been realized as well, measuring signals from strong electronic transition dipole moments in solids ${ }^{20-22}$ or rotational states in the gas-phase. ${ }^{23}$ The still rather limited available energies of $\mathrm{THz}$ pulses and the experimental difficulties in generation and recombination of multiple $\mathrm{THz}$ pulses, however, have not allowed yet the realization of such experiments in liquids, whose transition dipoles are extremely small. These challenges have set the stage for the development of experimental methods relying on hybrid pulse sequences. Bonn and coworkers, very recently, combined $\mathrm{THz}$, infrared and visible pulses to measure coupling between the high and low frequency modes in liquid water. ${ }^{24}$ In our group, hybrid 2D Raman-THz spectroscopy with a Raman-THz-THz (RTT) pulse sequence was developed ${ }^{25}$, and used to measure signals from water ${ }^{26}$ aqueous salt solutions ${ }^{27}$ and to 
disentangle the contribution of nuclear quantum effects to water's structure in different water isotopologues. ${ }^{28}$ Blake and coworkers have introduced the complementary $\mathrm{THz}-\mathrm{THz}-\mathrm{Raman}$ (TTR) pulse sequence and applied it to halogenated liquids. ${ }^{29,30}$ That pulse sequence extends the THz-Kerr-effect ${ }^{31-34}$ by separating the nonlinear $\mathrm{THz}$ interaction of the latter into two pulses.

Here, we report 2D RTT spectra measured for the same type of liquids, whose sharp intra-molecular modes serve as perfect model system for understanding the capabilities of this novel spectroscopic technique. The spectra reveal cross peaks, whose frequency positions, after proper treatment of the instrument response function, suggest strong coupling between the intra-molecular modes of the molecule and the broad inter-molecular degrees of freedom of the liquid. This result differs significantly from that of Blake and coworkers, who attributed cross peaks to couplings between two intramolecular modes of the molecule, suggesting that the two techniques are complementary to each other and are sensitive to different types of information. The ability of 2D Raman- $\mathrm{THz}$ spectroscopy of revealing such intra/inter-molecular couplings makes it a decisive tool to investigate in more detail the dynamics of collective molecular motion in the low-frequency regime for the liquid-phase.

Fig. 1a shows the 2D Raman-THz signal for liquid bromoform $\left(\mathrm{CHBr}_{3}\right)$, with the color scale saturated such that the oscillating signal stands out. As already outlined in previous publications, ${ }^{26,27}$ the delay lines' arrangement in our 2D Raman- $\mathrm{THz}$ experimental setup allows to generate two distinct pulse sequences: the Raman- $\mathrm{THz}-$ THz (RTT) and the THz-Raman-THz (TRT) sequences. Delay $t_{1}$ is defined as the time between the first (Raman for RTT sequence and $\mathrm{THz}$ for TRT sequence) and the second ( $\mathrm{THz}$ for the RTT and Raman for the TRT) excitation pulses, whereas $t_{2}$ as the time between the $\mathrm{THz}$ excitation pulse and the emitted $\mathrm{THz}$ field. The part of the experimental data around $t_{1}=t_{2}=0$ up to $t_{1}=t_{2}= \pm 0.7 \mathrm{ps}$ is where the two pump-pulses overlap in time. For the RTT quadrant, the signal extends significantly beyond that region along the $t_{1}$ direction, even beyond our measurement window, while being very short lived along $t_{2}$. This long-lived dynamics allows us to analyze the signal starting from a $t_{1}$ delay $>0.7 \mathrm{ps}$ (see black vertical dashed line in Fig. 1a), where we can safely neglect the contribution from the pulse overlap. Conversely, the signal stemming from the TRT quadrant is much shorter lived and confined within the region where the pulse overlap is not yet decayed; this fact renders the proper extraction of the signal from this quadrant very complicated, and we will not discuss the TRT pulse sequence for the remainder of this paper. Fig. 1b shows a $1 \mathrm{D}$ cut along $t_{1}$ delay where the signal intensity is the highest (i.e. $t_{2}=0.1 \mathrm{ps}$ ). The signal consists of an oscillating component atop of an exponential decay, the latter of which reflects the alignment of molecules along the polarization direction of the Raman pulse and their relaxation to equilibrium. A single exponential fit
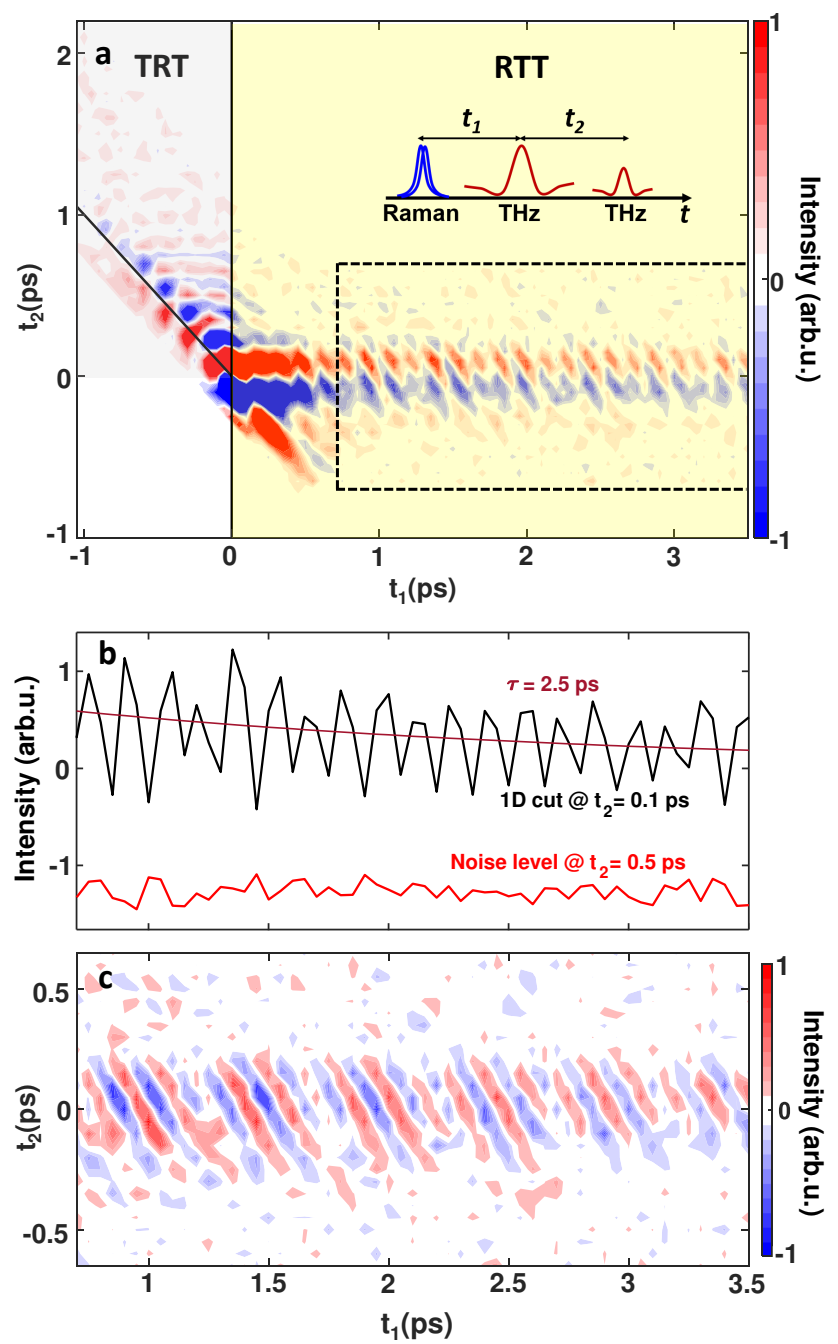

FIG. 1. (a) Experimental 2D Raman- $\mathrm{THz}$ data for bromoform; the region of the Raman- $\mathrm{THz}-\mathrm{THz}$ sequence is shaded in yellow, that of the THz-Raman-THz in grey. (b) $1 \mathrm{D}-\mathrm{Cut}$ of the signal for $t_{1} \geq 0.7$ ps at $t_{2}=0.1 \mathrm{ps}$. (c) Zoom of panel (a) with the exponential decay subtracted.

reveals a timescale of $\sim 2.5 \mathrm{ps}$, in agreement with the typical timescales found in optical Kerr effect (OKE) experiments for bromoform ${ }^{35}$ or related liquids. ${ }^{5,36,37} \mathrm{By}$ subtracting the single exponential fit from each 1D cut along $t_{1}$ direction, we can isolate the vibrational signal (Fig. 1c). Fig. 3a (see below) shows in red the absolutevalue of the $2 \mathrm{D}$ Fourier transformation of the data in Fig. 1c. Three strong peaks are clearly visible in the nonrephasing quadrant with $f_{1}, f_{2}>0$, labeled as $\mathrm{A}, \mathrm{A}^{\prime}$ and $\mathrm{B}$, together with a weaker one in the rephasing quadrant $\left(f_{1}<0, f_{2}>0\right)$, labeled as $\mathrm{C}$. The peaks are relatively narrow along the $f_{1}$-axis, where the frequency positions correspond to the well-known intramolecular vibrational modes $\nu_{6}\left(\sim 4.7 \mathrm{THz}\right.$ for peaks $\mathrm{A}, \mathrm{A}^{\prime}$ and $\left.\mathrm{C}\right)$ and $\nu_{3}$ ( $\sim 6.7 \mathrm{THz}$ for peak B) of bromoform. These peaks correspond to the doubly degenerate, asymmetric C-Br bend- 


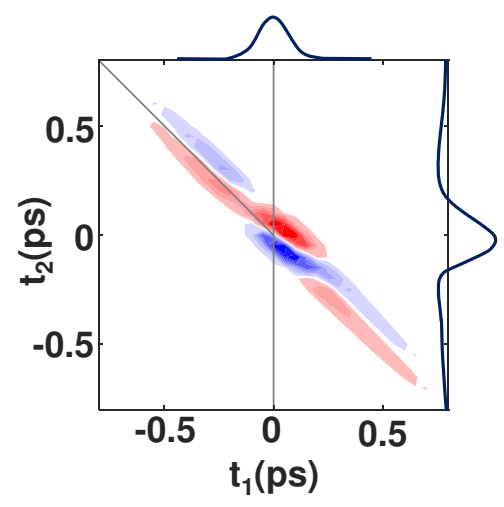

FIG. 2. IRF in the time-domain with the $\mathrm{THz}$ and Raman pulses shown along the right and upper axis.

ing mode and the symmetric $\mathrm{C}$-Br bending mode, respectively, and are seen both in one-dimensional Raman and THz spectra. ${ }^{29,38}$ Along the $f_{2}$-axis, peaks $\mathrm{A}$ and $\mathrm{B}$ are significantly broader with maxima at $\sim 3.5 \mathrm{THz}$. This frequency neither matches any of the fundamental modes of bromoform, nor difference frequencies of modes. Peak $\mathrm{C}$ in the rephasing part is about four times weaker than the non-rephasing bands and it peaks at a lower frequency (1.8 THz) along $f_{2}$.

To understand and assign this 2D Raman-THz spectrum, we have to consider very explicitly the instrument response function (IRF). That is, the molecular response function $R\left(t^{\prime \prime}, t^{\prime}\right)$ is convoluted with the not infinitesimally short excitation pulses: ${ }^{26}$

$$
\begin{aligned}
E^{(3)}\left(t_{1}, t_{2}\right)=\frac{d}{d t_{2}} & \int_{0}^{\infty} \int_{0}^{\infty} d t^{\prime} d t^{\prime \prime} E_{T H z}\left(t_{2}-t^{\prime \prime}\right) \\
& \cdot I_{\text {Raman }}\left(t_{2}+t_{1}-t^{\prime \prime}-t^{\prime}\right) R\left(t^{\prime \prime}, t^{\prime}\right),
\end{aligned}
$$

where $E_{T H z}$ is the field of the $\mathrm{THz}$ pulse and $I_{\text {Raman }}$ the intensity profile of the Raman pump pulse. This equation assumes that the mixing process is quasi phase-matched, and that absorption and dispersion effects in the propagation of the $\mathrm{THz}$ pulse from the sample to the detection are small (for a thorough discussion of these effects, see Ref. 26). We introduce the IRF:

$$
\operatorname{IRF}\left(t_{1}, t_{2}\right)=\frac{d}{d t_{2}}\left[E_{T H z}\left(t_{2}\right) \cdot I_{\text {Raman }}\left(t_{2}+t_{1}\right)\right]
$$

which is shown in Fig. 2. In that calculation, we used the $\mathrm{THz}$ pulse as measured during the collection of the 2D data, and an Gaussian-shaped intensity profile for the Raman pulse $I_{\text {Raman }}$ with a fitted FWHM of 110 fs (the pulses are shown along the right and upper axis in Fig. 2, respectively).

We can write Eq. 1 as a convolution (i.e., $\circledast)$ of the molecular response function $R\left(t^{\prime \prime}, t^{\prime}\right)$ with the IRF: ${ }^{39}$

$$
E^{(3)}\left(t_{1}, t_{2}\right)=\operatorname{IRF}\left(t_{1}, t_{2}\right) \circledast R\left(t_{1}, t_{2}\right) .
$$
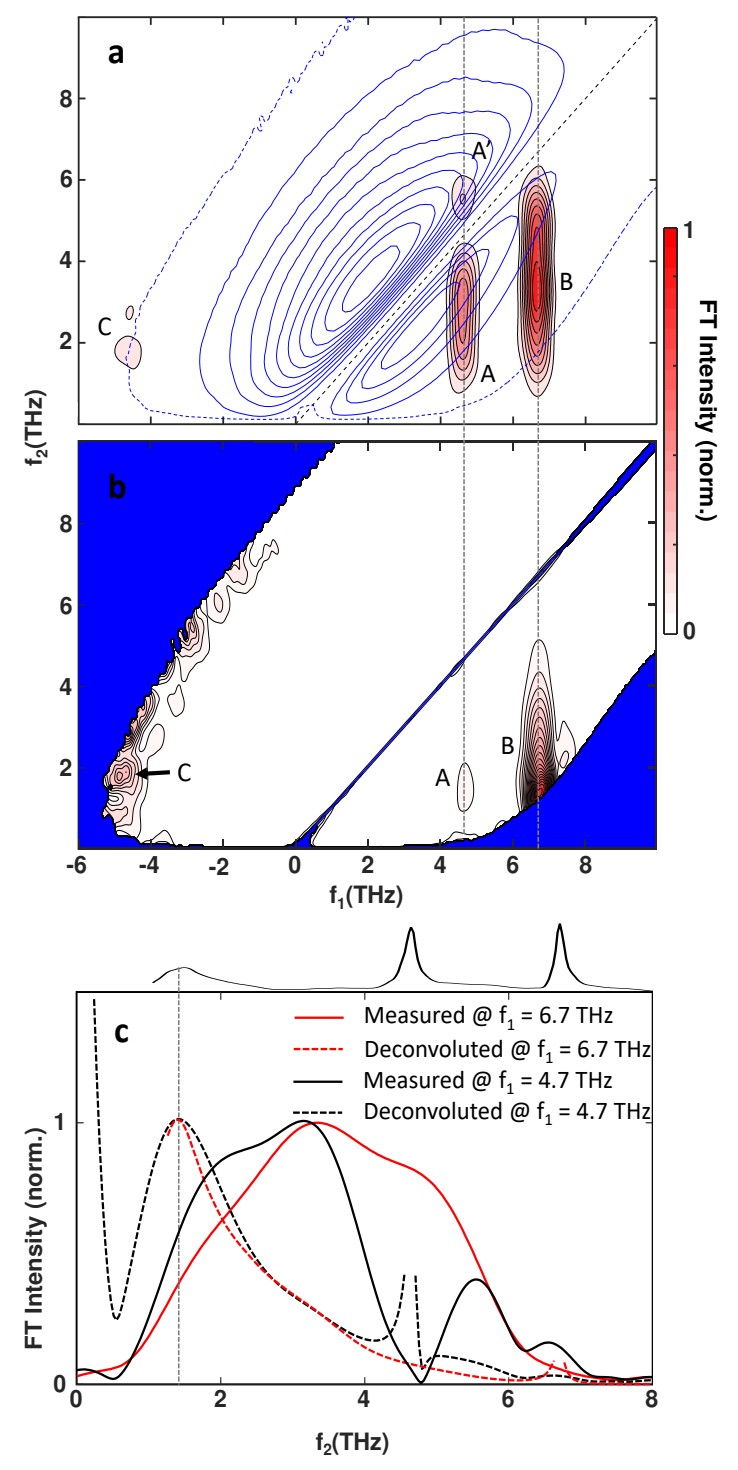

FIG. 3. (a) Absolute-value of the 2D Fourier transformation for the bromoform data in Fig. 1b (filled red contours) overlaid with that of the IRF (the blue solid contour lines are in steps of $10 \%$ of the maximum value, and the $1 \%$ contour line is added as blue-dashed line). (b) The bromoform data from panel (a) after deconvolution. The blue areas blank out those parts where the IRF is too small to trust the deconvolution, i.e. applying a threshold of $0.5 \%$ of the maximum amplitude of the IRF. The arrow marks peak $\mathrm{C}$ after deconvolution. (c) Measured (solid lines) and deconvoluted (dashed lines) 1D vertical cuts along the $f_{2}$-direction for $f_{1}$ corresponding to the fundamental intramolecular vibrations. The 1D-Raman spectrum of bromoform is shown atop of panel (digitized from Ref. 38).

Applying the convolution theorem:

$$
\tilde{E}^{(3)}\left(f_{1}, f_{2}\right)=I \tilde{R} F\left(f_{1}, f_{2}\right) \cdot \tilde{R}\left(f_{1}, f_{2}\right),
$$

we see that the $2 \mathrm{D}$ Raman-THz spectrum in the frequency domain becomes a product of the Fourier transformation of the molecular response function 
$\tilde{R}\left(f_{1}, f_{2}\right)$ with that of the instrument response function $I \tilde{R} F\left(f_{1}, f_{2}\right)$. The absolute-value of the latter is shown in Fig. 3a as blue contour lines. Most of its amplitude resides in the non-rephasing quadrant (i.e., $f_{1}, f_{2}>0$ ) and it consists of two lobes divided by a nodal line on the diagonal where $f_{1}=f_{2}$; another zero-amplitude line lies along the $f_{1}$ axis. It becomes evident from Fig. 3a how the spectral shape of the IRF is imprinted on the measured response, especially along the diagonal node (i.e. $f_{1}=f_{2}$ ) that "splits" the peak at $f_{1}=4.7 \mathrm{THz}$. In fact, the doublet $\mathrm{A}$ and $\mathrm{A}$ ' originate from one and the same peak, and $\mathrm{A}^{\prime}$ is just the extension of A above the diagonal line.

We can derive the "real" molecular response by dividing the measured spectrum through the IRF (Fig. 3b), thereby effectively deconvoluting the data. The deconvolution is defined only in certain parts of the spectrum, while regions, in which the IRF is too small for the deconvoloution to be reliable, have been blanked out in Fig. 3b (blue areas). Three conclusions can be drawn from the deconvoluted spectrum: First, $f_{1}$ of peaks A and $\mathrm{B}$ do not change; they still encode the fundamental intramolecular modes $\nu_{6}$ and $\nu_{3}$. Secondly, the relative intensities of these peaks change significantly, rendering the higher frequency peak B much more intense. Thirdly and most importantly, the peaks shifted significantly in $f_{2}$ towards lower frequencies with maxima now around $f_{2} \approx 1.3 \mathrm{THz}$. This shift becomes even more evident when plotting in Fig. $3 \mathrm{c} 1 \mathrm{D}$ cuts along the $f_{2}$-axis for $f_{1^{-}}$ positions that correspond to the fundamental modes, i.e., $f_{1}=4.7 \mathrm{THz}$ and $f_{1}=6.7 \mathrm{THz}$. Comparing these cuts with the 1D Raman spectrum (plotted atop of Fig. 3c), ${ }^{38}$ we conclude that they coincide with the broad shoulder that is commonly attributed to the intermolecular modes of the liquid at around $\sim 1.6 \mathrm{THz}$. The band is typically assigned to librational motions of the molecule that are seen universally in polar liquids. ${ }^{38,40}$ The cross peaks can thus be assigned to the coupling between the sharp intraand the much broader intermolecular modes of the liquid. This coupling is much stronger for the higher frequency mode $\nu_{3}$. Even though we do presently not understand why that might make a difference, we note that $\nu_{6}$ is a doubly degenerate mode, while $\nu_{3}$ is singly degenerate.

Peak $\mathrm{C}$ in the rephasing quadrant is situated very much at the edge where the deconvolution becomes unreliable (marked by an arrow Fig. 3b). We can nevertheless conclude that its $f_{2}$ peak position is less affected by the convolution with the IRF (we derived $1.8 \mathrm{THz}$ from Fig. 3a), since its position is close to the maximum of the IRF in the $f_{2}$-direction, i.e., since the $1 \%$ contour line (Fig. $3 \mathrm{a}$, dashed blue) crosses that peak vertically.

To second our interpretation of intra-/inter molecular coupling, we considered another halogenated molecule, diiodomethane $\left(\mathrm{CH}_{2} \mathrm{I}_{2}\right)$ with an even simpler $1 \mathrm{D}$ spectrum with only one intramolecular mode $\left(\nu_{4}\right.$ at $3.7 \mathrm{THz}$, the C-I bending mode) in our detection window. Applying the same procedure to the data as for bromoform, the measured and deconvoluted spectra are shown in Fig. 4a
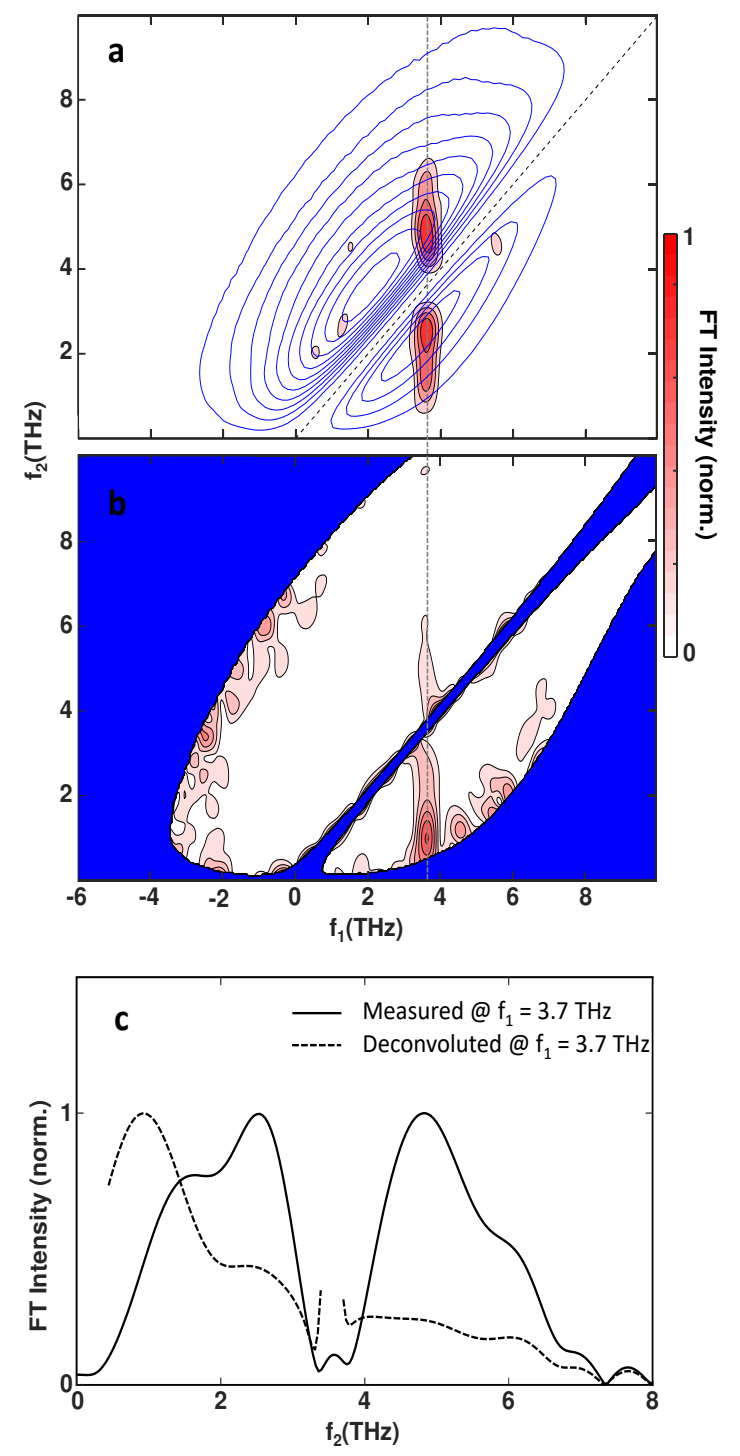

FIG. 4. Same as Fig. 3, but for diiodomethane with only one intramolecular mode in the accessible frequency range, i.e., (a) absolute-value 2D spectrum overlaid with the IRF, (b) the data from panel (a) after deconvolution, and (c) measured (solid line) and deconvoluted (dashed lines) 1D vertical cuts along the $f_{2}$-direction for $f_{1}$ corresponding to the fundamental intramolecular vibration. In panel (b), the threshold, above which the deconvolution was deemed to be reliable, was set to $5 \%$ of the maximum amplitude of the IRF.

and $\mathrm{b}$, respectively, and the $1 \mathrm{D}$ cuts along $f_{2}$ in Fig. $4 \mathrm{c}$. It can be seen again how strongly the IRF affects the data, splitting the peak into two parts. This effect is even more pronounced than for bromoform, since the mode is lower in frequency. Analyzing the the spectra before and after deconvolution, we arrive at the same conclusions as for bromoform: the peak position in $f_{2}$ is shifted downwards significantly upon deconvolution into a spectral region, in which intermolecular modes are expected (to our knowledge, the $1 \mathrm{D}$ spectrum of this molecule below $3 \mathrm{THz}$ is 
not present in literature).

Bromoform, among other molecules, has been investigated by Blake and coworkers with the TTR pulse sequence, ${ }^{29,30}$ while our experiment uses an inverted RTT pulse sequence. In either case, one of the interactions must induce a two-quantum transition, ${ }^{41}$ which would be forbidden in the harmonic approximation. In order to reveal a cross peak between two degrees of freedom, that two-quantum transition must be in the middle of the pulse sequence, de-exciting one mode and exciting another one at the same time. Both pulse sequences have a $\mathrm{THz}$ interaction in the middle, hence we conclude, just like Blake and coworkers, ${ }^{29,30}$ that the source of anharmonicity is mostly related to the dipole function of the system.

Based on their frequency positions, however, Blake and coworkers assigned a sequence of cross peaks to couplings between two intramolecular modes, involving thermally excited states, ${ }^{29,30}$ while we assign the one observed cross peak to intra/inter molecular couplings. The mechanical anharmonicities of the intramolecular modes of bromoform are very small, ${ }^{42}$ while one might expect that those of intermolecular degrees of freedom are significantly larger. Furthermore, the very fact that the $2 \mathrm{D}$ Raman-THz spectrum of diiodomethane shows the same cross peak rules out any possible intra-/intra molecular coupling, as that molecule has only one mode $\left(\nu_{4}\right)$ within the frequency window of our experiment. The next higher mode, $\nu_{3}$ at $\approx 15 \mathrm{THz},{ }^{43}$ is clearly beyond that frequency range, and thermal excitation becomes small $(<10 \%)$ as well. Apparently, the two techniques, THz-THz-Raman vs Raman-THz-THz-Raman, are complementary, going through different coherence pathways, and are sensitive to a different set of anharmonic couplings.

In conclusion, we have measured the 2D Raman- $\mathrm{THz}$ spectrum of bromoform and diiodomethane, providing evidence that the observed cross peaks are a signature of coupling between the narrow intramolecular modes of the molecules and the broad continuum related to the intermolecular degrees of freedom of the liquid. The fact that the signal is highly convoluted with the IRF requires a careful analysis in order to extract the real positions of the peaks and in turn the information that these peaks carry. The non-intuitive and complex IRF is related to the intricate shape of $\mathrm{THz}$ pulse and therefore it is crucial to take into account the convolution each time the experiments involve one or more $\mathrm{THz}$ excitations. The details of the anharmonic coupling between intraand intermolecular modes are, however, not known at this point and will require significant attention from the theory side. The TTR pulse sequence used by Blake and coworkers ${ }^{29,30}$ and our RTT pulse sequence share a nonlinear $\mathrm{THz}$ interaction in the middle as the one that gives rise to cross peaks, while the third pulse sequence, TRT, does that via a Raman-interaction. Proper extraction of that response function will reveal yet another set of anharmonic couplings in these molecular liquids.
Methods: All measurement were performed essentially with the same setup as in Refs. 26-28. Briefly, the output of a Ti:Sapphire laser $(5 \mathrm{kHz}, 0.8 \mathrm{~mJ} /$ pulse, $\sim 100$ fs duration) is split into three branches: Raman pump, $\mathrm{THz}$ generation and $\mathrm{THz}$ detection. The $\mathrm{THz}$ pulses, whose spectrum extends up to $7 \mathrm{THz}$, are generated via optical rectification in a $100 \mu \mathrm{m}$ thick GaP crystal, subsequently focused on the sample and then on the detection crystal (an equivalent $100 \mu \mathrm{m}$ thick GaP crystal) by means of two custom-made elliptical aluminum mirrors. The $\mathrm{THz}$ electric field is measured via electro-optic sampling. The delay $t_{1}$ between the Raman pump and the $\mathrm{THz}$ pulse is controlled with a conventional delay stage on the Raman pump branch. The sampling delay $t_{2}$, is scanned with a fast-scan motor (APE ScanDelay) on the $\mathrm{THz}$ detection branch. The foci of both $\mathrm{THz}$ and Raman pump pulses are matched on the sample position to a size of $\sim 250 \mu \mathrm{m}$. For the sample cell, we employ a sealed static cuvette made of two thin $(\sim 100 \mu \mathrm{m})$ sapphire windows separated by a $500 \mu \mathrm{m}$ teflon spacer for bromoform and $200 \mu \mathrm{m}$ for diiodomethane. The energy of Raman pulses was set to $\sim 7 \mu \mathrm{J}$ to avoid contribution from pump-induced nonlinear processes in the sample. Gaussian apodization has been applied to all time domain data. The averaging time of each measurement amounts to about 4 days. All the measurements were performed at room temperature.

Acknowledgement: We thank Geoffrey Blake and Thomas Miller for very interesting discussions and for sending us a draft prior to publication of their most recent work on the theoretical assignment of their results. We also thank David Sidler for valuable discussions, who actually introduced the idea to calculate the IRF in the frequency domain in Ref. 39, which has been the key for the understanding of the present data. The work has been supported by the Swiss National Science Foundation (SNF) through the National Center of Competence and Research (NCCR) MUST.

\section{References:}

(1) Bagchi, B.; Jana, B. Solvation dynamics in dipolar liquids, Chem. Soc. Rev. 2010, 39, 1936-1954.

(2) Horng, M. L.; Gardecki, J. A.; Papazyan, A.; Maroncelli, M. Subpicosecond measurements of polar solvation dynamics: Coumarin 153 revisited, J. Phys. Chem. 1995, 99, 17311-17337.

(3) Heisler, I. A.; Meech, S. R. Low-frequency modes of aqueous alkali halide solutions: Glimpsing the hydrogen bonding vibration, Science 2010, 327, 857-860.

(4) Fecko, C. J.; Eaves, J. D.; Tokmakoff, A. Isotropic and anisotropic Raman scattering from molecular liquids measured by spatially masked optical Kerr effect spectroscopy, J. Chem. Phys 2002, 117, 1139-1154.

(5) Loughnane, B. J.; Scodinu, A.; Farrer, R. A.; Fourkas, J. T.; Mohanty, U. Exponential intermolecular dynamics in optical Kerr effect spectroscopy of small-molecule liquids, J. Chem. Phys. 1999, 111, 2686-2694. 
(6) Fukasawa, T.; Sato, T.; Watanabe, J.; Hama, Y.; Kunz, W.; Buchner, R. Relation between dielectric and low-frequency Raman spectra of hydrogen-bond liquids, Phys. Rev. Lett. 2005, 95, 197802.

(7) Meister, K.; Ebbinghaus, S.; Xu, Y.; Duman, J. G.; DeVries, A.; Gruebele, M.; Leitner, D. M.; Havenith, M. Long-range protein-water dynamics in hyperactive insect antifreeze proteins, Proc. natl. Acad. Sci. USA 2013, 110, 1617-1622.

(8) Ernst, R.; Bodenhausen, G.; Wokaun, A. Principles of Nuclear Magnetic Resonance in One and Two Dimensions; Clarendon Press, Oxford, 1987.

(9) Hamm, P.; Zanni, M. Concepts and methods of $2 D$ infrared spectrsocopy; Cambridge University Press, Cambridge, 2011.

(10) Hamm, P.; Lim, M.; Hochstrasser, R. M. Non-markovian dynamics of the vibrations of ions in water from femtosecond infrared three-pulse photon echoes, Phys. Rev. Lett. 1998, 81, 5326-5329.

(11) Tokmakoff, A.; Fayer, M. D. Homogeneous vibrational dynamics and inhomogeneous broadening in glassforming liquids: Infrared photon echo experiments from room temperature to $10 \mathrm{~K}$, J. Chem. Phys. 1995, 103, 2810-2826.

(12) Lu, J.; Li, X.; Zhang, Y.; Hwang, H. Y.; Ofori-Okai, B. K.; Nelson, K. A. Two-dimensional spectroscopy at terahertz frequencies, Top. Curr. Chem. 2018, 376.

(13) Tanimura, Y.; Mukamel, S. Two-dimensional femtosecond vibrational spectroscopy of liquids, J. Chem. Phys. 1993, 99, 9496-9511.

(14) Golonzka, O.; Demirdöven, N.; Khalil, M.; Tokmakoff, A. Separation of cascaded and direct fifth-order Raman signals using phase-sensitive intrinsic heterodyne detection, J. Chem. Phys. 2000, 113, 9893-9896.

(15) Tokmakoff, A.; Lang, M. J.; Larsen, D. S.; Fleming, G. R.; Chernyak, V.; Mukamel, S. Two-dimensional Raman spectroscopy of vibrational interactions in liquids, Phys. Rev. Lett. 1997, 79, 2702-2705.

(16) Blank, D. A.; Kaufman, L. J.; Fleming, G. R. Direct fifth-order electronically nonresonant Raman scattering from cs2 at room temperature, J. Chem. Phys. 2000, 113, 771-778.

(17) Kubarych, K.; Milne, C.; Miller, R. Heterodyne detected fifth-order Raman response of liquid CS2: Dutch cross polarization, Chem. Phys. Lett. 2003, 369, 635 - 642.

(18) Li, Y. L.; Huang, L.; Dwayne Miller, R. J.; Hasegawa, T.; Tanimura, Y. Two-dimensional fifth-order Raman spectroscopy of liquid formamide: Experiment and theory, J. Chem. Phys. 2008, 128, 234507.

(19) Frostig, H.; Bayer, T.; Dudovich, N.; Eldar, Y. C.; Silberberg, Y. Single-beam spectrally controlled twodimensional Raman spectroscopy, Nat. Phot. 2015, 9, 339-343.

(20) Kuehn, W.; Reimann, K.; Woerner, M.; Elsaesser, T.; Hey, R. Two-dimensional terahertz correlation spectra of electronic excitations in semiconductor quantum wells, J. Phys. Chem. B 2011, 115, 5448-5455.

(21) Kuehn, W.; Reimann, K.; Woerner, M.; Elsaesser, T. Phase-resolved two-dimensional spectroscopy based on collinear n-wave mixing in the ultrafast time domain, $J$. Phys. Chem. 2009, 130, 164503.

(22) Elsaesser, T.; Reimann, K.; Woerner, M. Concepts and Applications of Nonlinear Terahertz Spectroscopy; Morgan and Claypool Publishers, 2019.
(23) Lu, J.; Zhang, Y.; Hwang, H. Y.; Ofori-Okai, B. K.; Fleischer, S.; Nelson, K. A. Nonlinear two-dimensional terahertz photon echo and rotational spectroscopy in the gas phase, Proc. Natl. Acad. Sci. USA 2016, 113, 1180011805.

(24) Grechko, M.; Hasegawa, T.; D’Angelo, F.; Ito, H.; Turchinovich, D.; Nagata, Y.; Bonn, M. Coupling between intra- and intermolecular motions in liquid water revealed by two-dimensional terahertz-infrared-visible spectroscopy, Nat. Comm. 2018, 9, 885.

(25) Hamm, P.; Savolainen, J. Two-dimensional-Ramanterahertz spectroscopy of water: Theory, J. Chem. Phys. 2012, 136, 094516.

(26) Savolainen, J.; Ahmed, S.; Hamm, P. Two-dimensional Raman-terahertz spectroscopy of water, Proc. Natl. Acad. Sci. USA 2013, 110, 20402-20407.

(27) Shalit, A.; Ahmed, S.; Hamm, P. Terahertz echoes reveal the inhomogeneity of aqueous salt solutions, Nat. Chem. 2017, 9, 273-278.

(28) Berger, A.; Ciardi, G.; Sidler, D.; Hamm, P.; Shalit, A. Impact of nuclear quantum effects on the structural inhomogeneity of liquid water, Proc. Natl. Acad. Sci. USA 2019, 116, 2458-2463.

(29) Finneran, I. A.; Welsch, R.; Allodi, M. A.; Miller, T. F.; Blake, G. A. Coherent two-dimensional terahertzterahertz-Raman spectroscopy, Proc. Natl. Acad. Sci. USA 2016, 113, 6857-6861.

(30) Finneran, I. A.; Welsch, R.; Allodi, M. A.; Miller, T. F.; Blake, G. A. 2D THz-THz-Raman photon-echo spectroscopy of molecular vibrations in liquid bromoform, J. Phys. Chem. Letter 2017, 8, 4640-4644.

(31) Allodi, M. A.; Finneran, I. A.; Blake, G. A. Nonlinear terahertz coherent excitation of vibrational modes of liquids, J. Chem. Phys. 2015, 143, 234204.

(32) Hoffmann, M. C.; Brandt, N. C.; Hwang, H. Y.; Yeh, K.-L.; Nelson, K. A. Terahertz Kerr effect, Appl. Phys. Lett. 2009, 95, 231105.

(33) Sajadi, M.; Wolf, M.; Kampfrath, T. Transient birefringence of liquids induced by terahertz electric-field torque on permanent molecular dipoles, Nat. Comm. 2017, 8, 14963.

(34) Kampfrath, T.; Campen, R. K.; Wolf, M.; Sajadi, M. The nature of the dielectric response of methanol revealed by the terahertz Kerr effect, J. Chem. Phys. Lett. 2018, 9, 1279-1283.

(35) Shirota, H.; Kato, T. Intermolecular vibrational spectra of C3v CXY3 molecular liquids, CHCl3, CHBr3, CFBr3, and $\mathrm{CBrCl} 3$, J. Phys. Chem. A 2011, 115, 8797-8807.

(36) Hunt, N. T.; Jaye, A. A.; Meech, S. R. Ultrafast dynamics in complex fluids observed through the ultrafast optically-heterodyne-detected optical-Kerr-effect (OHDOKE), Phys. Chem. Chem. Phys. 2007, 9, 2167-2180.

(37) Wiewiór, P. P.; Shirota, H.; Castner, E. W. Aqueous dimethyl sulfoxide solutions: Inter- and intra-molecular dynamics, J. Chem. Phys. 2002, 116, 4643-4654.

(38) Afsar, M.; Hasted, J.; Zafar, M.; Chamberlain, J. Absorption bands in liquid chloroform and bromoform, Chem. Phys. Lett. 1975, 36, $69-72$.

(39) Sidler, D.; Hamm, P. Feynman diagram description of 2D-Raman- $\mathrm{THz}$ spectroscopy applied to water, J. Chem. Phys. 2019, 150, 044202.

(40) Davies, M.; Pardoe, G. W. F.; Chamberlain, J. E.; Gebbie, H. A. Character of absorption in far infra-red by polar molecules in liquid state, Trans. Faraday Soc. 1968, 
$64,847-860$.

(41) Hamm, P.; Shalit, A. Perspective: Echoes in 2D-RamanTHz spectroscopy, J. Chem. Phys. 2017, 146, 130901.

(42) Fernández-Liencres, M. P.; Navarro, A.; López, J. J.; Fernández, M.; Szalay, V.; de los Arcos, T.; GarcíaRamos, J. V.; Escribano, R. M. The force field of bro- moform: A theoretical and experimental investigation, J. Chem. Phys. 1996, 100, 16058-16065.

(43) Ford, T. Infrared and Raman spectra and vibrational assignments of diiodomethane and its deuterated analogs, J. Mol. Spec. 1975, 58, $185-193$. 